Characterization of mutations and sequence variants in the D21S11 locus by next generation sequencing

Rockenbauer, Eszter; Hansen, Stine; Mikkelsen, Martin; Børsting, Claus; Morling, Niels

Published in:

Forensic science international. Genetics

DOI:

10.1016/j.fsigen.2013.06.011

Publication date:

2014

Citation for published version (APA):

Rockenbauer, E., Hansen, S., Mikkelsen, M., Børsting, C., \& Morling, N. (2014). Characterization of mutations and sequence variants in the D21S11 locus by next generation sequencing. Forensic science international. Genetics, 8(1), 68-72. https://doi.org/10.1016/j.fsigen.2013.06.011 


\title{
Characterization of mutations and sequence variants in the D21S11 locus by next generation sequencing
}

\author{
Eszter Rockenbauer ${ }^{\mathrm{a}, 1, *}$, Stine Hansen ${ }^{\mathrm{b}, 1}$, Martin Mikkelsen ${ }^{\mathrm{a}}$, Claus Børsting ${ }^{\mathrm{a}}$, \\ Niels Morling a \\ ${ }^{a}$ Section of Forensic Genetics, Department of Forensic Medicine, Faculty of Health and Medical Sciences, University of Copenhagen, Denmark \\ ${ }^{\mathrm{b}}$ Centre of Forensic Genetics, Department of Medical Biology, Faculty of Health Sciences, University of Tromsø, Norway
}

\section{A R T I C L E I N F O}

\section{Article history:}

Received 25 January 2013

Received in revised form 14 June 2013

Accepted 18 June 2013

\section{Keywords:}

Next generation sequencing

Short tandem repeat

Genetic inconsistency

Mutation

Paternity case

\begin{abstract}
A B S T R A C T
We sequenced the D21S11 locus in 77 individuals from Danish paternity cases using 454 FLX next generation sequencing (NGS) technology. All samples were also typed with the AmpFlSTR ${ }^{\circledR}$ Profiler Plus ${ }^{\circledR}$ or the AmpFlSTR ${ }^{\mathbb{R}}$ Identifiler ${ }^{\mathbb{R}}$ PCR Amplification kits as part of paternity investigations. In 18 of the confirmed trios, a genetic inconsistency was observed between one of the parents and the child at the D21S11 locus. NGS of the D21S11 locus revealed which allele had mutated from which parent to the child in 13 of these trios. All characterized mutations could be explained by single-step mutations in the longest sub-repeat of D21S11. A total of 53 of the 77 sequenced samples originated from unrelated individuals. Twenty different D21S11 alleles were detected by NGS in these individuals whereas only 13 different alleles were observed with fragment analysis. Several alleles had the same lengths but different sequences, e.g. four and three different alleles were detected by NGS with lengths determined by CE corresponding to allele 30 and allele 31 , respectively.
\end{abstract}

(c) 2013 Elsevier Ireland Ltd. All rights reserved.

\section{Introduction}

Fragment analysis of STR loci by PCR and capillary electrophoresis (CE) is presently the preferred tool for STR profiling in forensic genetics. However, it was recently shown that next generation sequencing (NGS) technologies are well suited for STR sequencing and may be the first real alternative to large scale CE-based STR typing [1-4]. Two different NGS methods were investigated, 454 FLX pyrosequencing (Roche Diagnostics) and reversible terminator-based sequencing (Illumina). Both technologies were superior in revealing sequence variation compared to standard fragment analysis on CE platforms. The 454 FLX pyrosequencing allows sequencing of longer fragments (5-600 bp), than Illumina's reversible terminator-based sequencing (150 bp) [2,5]. However, the error rate, especially at homopolymer stretches, is considerably higher with the 454 FLX pyrosequencing than with the Illumina method [5]. Electrospray ionization mass spectrometry (ESI-MS) has also been used successfully to distinguish between STR alleles

\footnotetext{
* Corresponding author at: Section of Forensic Genetics, Department of Forensic Medicine, Faculty of Health and Medical Sciences, University of Copenhagen, Frederik V's Vej 11, DK-2100 Copenhagen, Denmark. Tel.: +45 353261 10; fax: +4535326120 .

E-mail addresses: eszter.rockenbauer@forensic.ku.dk, rockenbauer@hotmail.com (E. Rockenbauer).

${ }^{1}$ These authors contributed equally to this manuscript.
}

with equal lengths [6,7]. However, with ESI-MS, it is only possible to determine the basepair composition and not the sequence. Thus, alleles with the same length and the same base pair composition, but with different sequences, cannot be distinguished from each other. Mutations in Y STR loci have been investigated by Sanger sequencing in a number of studies [8-11]. However, there are only a few publications on characterization of mutations in autosomal STR loci [12-14] most likely because it required sequencing of isolated clones prior to the development of emulsion PCR and clonal sequencing. Complex STR loci, such as D21S11, D2S1338 and D3S1358, consist of interrupted repeat sequences. Variations in repeat numbers may be observed in more than one sub-repeat unit within the loci, which makes these STR systems good candidates for sequence variation studies. The average mutational rates of the core STR loci are estimated to be between $0.01 \%$ and $0.64 \%$ (http:// www.cstl.nist.gov/strbase/mutation.htm). However, the exact mutation rate of a locus seems to be associated with the base composition of the repeats and the length of the allele [15,16]. Furthermore, paternal mutations are more frequent than maternal ones [17-19], and single step mutations (involving the loss or gain of a single repeat unit) are more common than multi step mutations $[15,16]$. It is generally accepted, that mutations in STR loci are mainly caused by DNA polymerase slippage, where one or more repeat units are gained or lost [20]. However, other mechanisms including unequal crossing-over and gene conversions may also cause mutations [19]. 
D21S11 is a complex STR system with six sub-repeat sections and short interconnecting sequences. The known allele range is from 12 to 41.2 repeats. Variants with equal lengths but different sub-repeat compositions have been reported (http:// www.cstl.nist.gov/strbase/str_D21S11.htm [1,3]).

The aims of this study were to study (1) the sequence variation and allele frequencies of D21S11 alleles in unrelated Danes and (2) the mutations in D21S11 by sequencing parent-child pairs with genetic inconsistencies in confirmed trios in Danish paternity cases.

\section{Materials and methods}

\subsection{Samples}

The work was approved by the Danish ethical committee (H-12011-081). The samples originated from 77 individuals in 29 Danish paternity cases. A total of 22 of these cases were confirmed trios, 1 case was a mother-child duo, and 1 case was a father-child duo. In the last five cases, samples from seven unrelated persons were selected. Of the 77 sequenced samples, 53 originated from unrelated individuals. The samples were collected as either buccal swabs or anticoagulated blood. The individuals were typed with either the AmpFlSTR ${ }^{\circledR}$ Profiler Plus ${ }^{\circledR}$ kit or the AmpFlSTR ${ }^{\circledR}$ Identifiler ${ }^{\circledR}$ PCR Amplification kit (Applied Biosystems/Life Technologies, Foster City, CA, USA) during the initial casework investigation. Supplementary investigations of either 5-7 VNTR loci [21] or 49 SNPs [22] were performed in cases with one genetic inconsistency in D21S11. No additional inconsistencies were observed and the combined PI was higher than 10,000 in all cases. In three trios, a three-allelic pattern was observed in either the child (two cases) or the mother (one case). Additionally, one sample from a male with three alleles in D21S11 was included.

\subsection{Extraction and $P C R$ amplification}

DNA was isolated manually with the QIAmp ${ }^{\circledR}$ DNA Blood Mini Kit (Qiagen, Hilden, Germany) or automatically by using a EZ1 Advanced XL BioRobot (Qiagen) as recommended by the manufacturer. A total of 30 fusion primers with multiplex identifers (MIDs) were designed according to the protocol "454 Sequencing System Guidelines for Amplicon Experimental Design" (Roche Diagnostics, Basel, Switzerland, July 2011) and Kline et al. [23]. The sequences of all fusion primers are presented in Supplementary Table S1. The samples were amplified as previously described [1]. PCR products were evaluated with the Flashgel System (Lonza, Rockland, ME, USA). Excess primers and nucleotides were removed using the Agencourt AMPure XP PCR Purification system (Beckman Coulter, Brea, CA, USA) following the guidelines of the manufacturer.

Supplementary data associated with this article can be found, in the online version, at doi:10.1016/j.fsigen.2013.06.011.

\subsection{Sample quantification}

The samples were quantified using the QuantiFlour ${ }^{\mathrm{TM}} \mathrm{dsDNA}$ System Kit (Promega, Fitchburg, WI, USA). Standard curves were created using the Lambda DNA Standard supplied with the kit. Sample concentrations were calculated following the guidelines of the manufacturer.

\subsection{FLX pyrosequencing}

The samples were sequenced using the GS Junior System (Roche Diagnostics). Three sequencing runs were carried out. The output data was sorted as previously described [1]. An algorithm sorted the sequencing data according to MID-tags, primer sequences and STR-ends, and created an output file containing sequences for each sample. Incomplete STR sequences were removed. Sequences were aligned toward the D21S11 reference sequence with 29 repeats (GenBank AP000433) using the alignment software BioEdit (URL: http://www.mbio.ncsu.edu/bioedit/bioedit.html).

\subsection{Capillary electrophoresis}

All samples were typed with the AmpF $\ell$ STR ${ }^{\circledR}$ Identifiler $^{\circledR}$ Direct PCR Amplification Kit according to ISO 17025 accredited protocols as previously described [24,25].

\section{Results}

\subsection{Sequencing data}

Three sequencing runs resulted in a total of 192,011 reads. Only sequences containing primer sequences and the entire STR repeat sequence of D21S11 were analyzed further [1]. An average of 147 reads per sample (range: $26-220$ reads) was obtained. Sequences of each allele were grouped according to repeat region lengths and aligned to a reference sequence of D21S11 with 29 repeats (GenBank AP000433). Allele calls were computed and compared to those determined by the AmpF $\ell S T R^{\circledR}{ }^{\circledR}$ Identifiler ${ }^{\circledR}$ Direct kit (see Supplementary Table S2, for allele calls and numbers of sequence reads.) There was complete concordance between the allele calls determined by 454 FLX pyrosequencing and CE. The ratios between the allele represented with the fewest reads and the allele represented with the most reads in a sample varied from 0.26 to 1 , with an average of 0.71 (Supplementary Table S3). Generally, fewer reads were observed for longer STR alleles. The longest allele had 36 repeats. The total number of reads for this sample was 37 (including stutter reads). The ratio between the numbers of reads representing the two alleles (33.2 and 36) was 0.62. Even though longer sequences were somewhat underrepresented in the sequencing results, there were sufficient reads to unambiguously determine each allele. PCR artifacts including stutters (artifacts one repeat unit shorter or longer than the parent allele) were also observed in the sequencing data. Stutters made up approximately $15 \%$ of the sequence reads (data not shown), which is comparable with earlier results [1,3]. Amplification errors were mainly due to substitutions, deletions and insertions of single nucleotides and appeared, on average, in $26 \%$ of the sequences (data not shown).

Supplementary data associated with this article can be found, in the online version, at doi:10.1016/j.fsigen.2013.06.011.

\subsection{Characterization of mutations}

A total of 54 of the successfully sequenced samples came from individuals in 18 confirmed trios, where CE had demonstrated a genetic inconsistency at D21S11 between a parent and the child. The CE results suggested that a paternal or maternal single-step mutation could have occurred in nine and three cases, respectively. In the remaining six cases, either of the parents could have passed on a single-step mutation to the child. Under the assumption that only one mutation occurred at the D21S11 locus during meiosis, the sequencing results showed which parental allele most likely mutated in 13 of the 18 trios (Supplementary Table S4). In all families, the mutation most likely occurred in the longest subrepeat section of the STR system. The number of uninterrupted repeat units in the alleles, where the mutations were assumed to have arisen, ranged from 10 and 14 . The mutations could be characterized as either insertions (14 cases) or deletions ( 3 cases) of single repeat units. However, multi-step mutations could have happened in trio 7 and 14 (S4). One insertion of three and two 
Table 1

Trio 19 and 20 with three-allelic pattern in the child.

\begin{tabular}{|c|c|c|c|c|c|}
\hline Trio & & Sequence allele 1 & Sequence allele 2 & Sequence allele 3 & NGS phenotype \\
\hline \multirow[t]{3}{*}{ Trio 19} & Child & $\begin{array}{l}{[\text { TCTA }] 4[\text { TCTG]6[TCTA]3TA[TCTA]- }} \\
\text { 3TCA[TCTA]2TCCATA[TCTA] } 10\end{array}$ & $\begin{array}{l}{[\text { TCTA }] 4[\text { TCTG }] 6[\text { TCTA }] 3 T A[T C T A]-} \\
\text { 3TCA[TCTA }] 2 \text { TCCATA[TCTA }] 12\end{array}$ & 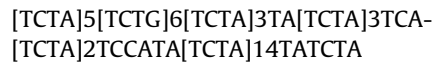 & $28 / 30 / 32.2$ \\
\hline & Mother & $\begin{array}{l}\text { [TCTA]4[TCTG]6[TCTA]3TA[TCTA]- } \\
3 \text { 3CA[TCTA]2TCCATA[TCTA]12 }\end{array}$ & $\begin{array}{l}\text { [TCTA]5[TCTG]6[TCTA]3TA[TCTA]- } \\
\text { 3TCA[TCTA]2TCCATA[TCTA]14TATCTA }\end{array}$ & & $30 / 32.2$ \\
\hline & Father & $\begin{array}{l}{[\text { TCTA }] 5[\text { TCTG }] 6[\text { TCTA]3TA[TCTA]- }} \\
\text { 3TCA[TCTA]2TCCATA[TCTA] } 11\end{array}$ & $\begin{array}{l}\text { [TCTA]5 [TCTG]6 [TCTA]3 TA [TCTA]- } \\
3 \text { TCA [TCTA]2 TCCATA [TCTA] } 11\end{array}$ & & $28 / 30$ \\
\hline \multirow[t]{3}{*}{ Trio 20} & Child & $\begin{array}{l}{[\text { TCTA }] 4[\text { TCTG]6[TCTA]3TA[TCTA]- }} \\
\text { 3TCA[TCTA]2TCCATA[TCTA]10 }\end{array}$ & $\begin{array}{l}\text { [TCTA]6[TCTG]5[TCTA]3TA[TCTA]- } \\
\text { 3TCA[TCTA]2TCCATA[TCTA]10 }\end{array}$ & $\begin{array}{l}\text { [TCTA }] 5[\text { TCTG]6[TCTA]3TA[TCTA]3TCA- } \\
\text { [TCTA]2TCCATA[TCTA]15TATCTA }\end{array}$ & $28 / 29 / 33.2$ \\
\hline & Mother & $\begin{array}{l}{[\text { TCTA }] 4[\text { TCTG }] 6[\text { TCTA]3TA[TCTA]- }} \\
\text { 3TCA[TCTA]2TCCATA[TCTA] } 10\end{array}$ & $\begin{array}{l}\text { [TCTA]5[TCTG]6[TCTA]3TA[TCTA]- } \\
\text { 3TCA[TCTA]2TCCATA[TCTA]15TATCTA }\end{array}$ & & $28 / 33.2$ \\
\hline & Father & $\begin{array}{l}\text { [TCTA]6[TCTG]5[TCTA]3TA[TCTA]- } \\
\text { 3TCA[TCTA]2TCCATA[TCTA] } 10\end{array}$ & $\begin{array}{l}\text { [TCTA]6 [TCTG]5 [TCTA]3 TA [TCTA]- } \\
3 \text { TCA [TCTA]2 TCCATA [TCTA]11 }\end{array}$ & & $29 / 30$ \\
\hline
\end{tabular}

repeats, respectively, may explain the inconsistencies (S4). In one family (trio 12), it was not possible to tell if the mutation was a deletion or an insertion, because of the similar sub-repeat pattern of the parent alleles.

Supplementary data associated with this article can be found, in the online version, at doi:10.1016/j.fsigen.2013.06.011.

\subsection{Samples with three alleles}

Four of the 77 sequenced samples had three-allelic patterns at D21S11. In three of the samples, the peek heights were of equal sizes, suggesting partial trisomy of the chromosomal area at the D21S11 locus (Supplementary Table S2). In the fourth sample, the sample of the mother from trio 21, allele 28 had a peek height equal to the sum of the peek heights of the other two alleles, alleles 30 and 33.2. The latter can be due to a somatic mutation in the D21S11 locus. With 454 FLX pyrosequencing, the three alleles were confirmed in all four samples. In trios 19 and 20, three-allelic patterns were observed in the children. Sequencing showed that two of the children's three alleles were in both cases identical to those of the mothers, which strongly suggests that both mothers passed on two alleles to the children (Table 1).

\subsection{Sequence variations in unrelated individuals}

The D21S11 locus was sequenced in 53 unrelated individuals (Table 2). A total of 20 different D21S11 alleles were identified by
454 FLX pyrosequencing, whereas only 13 different alleles were observed with capillary electrophoresis. Interestingly, sequencing data revealed that four of the individuals were heterozygous and not homozygous at the D21S11 locus. These individuals had two different alleles with the same lengths but with different subrepeat composition (Fig. 1).

The sequence of D21S11 can be written as [TCTA $]^{\mathrm{a}}[\mathrm{TCTG}]^{\mathrm{b}}[\mathrm{TC}-$ TA $]^{\mathrm{c}}$ TA[TCTA $]^{\mathrm{d}}$ TCA[TCTA $]^{\mathrm{e}}$ TCCATA[TCTA $]^{\mathrm{f}}$, where the superscripts refer to variation in repeat number. In the sequenced samples, we did not see any variation in the interconnecting sequences or in sub-repeats c, $d$ and e. The variations in sub-repeats $a, b$ and $f$ are shown in Table 3. The sequences of three of the 104 alleles are not in STRBase (http://www.cstl.nist.gov/div831/strbase/). Sequence variations were observed in many alleles of the same lengths (Table 2). Four different alleles with 30 repeats and three different alleles with 31 repeats were observed. Also, two different subrepeat composition of allele 29 and 30.2 were observed. This suggests a large variation in sub-repeat patterns in the Danish population.

\section{Discussion}

All the characterized mutations in this study could be explained by single-step mutations. However, in two cases, multi-step mutations may have taken place. Although multi-step mutations are considered to be rare, they cannot be disregarded [9,26,27]. The preferred direction of the STR mutations (insertion versus

Table 2

DNA sequences of D21S11 alleles and allele calls of the sequenced samples.

\begin{tabular}{|c|c|c|c|c|c|}
\hline $\begin{array}{l}\text { No. of alleles } \\
\text { all/European }\end{array}$ & Allele sequence & $\begin{array}{l}\text { Allele } \\
\text { call }\end{array}$ & $\begin{array}{l}\text { Total } \\
\text { frequency }\end{array}$ & $\begin{array}{l}\text { European } \\
\text { frequency }\end{array}$ & $\begin{array}{l}\text { In-house database } \\
\text { frequency }^{\mathrm{a}}\end{array}$ \\
\hline $1 / 0^{\mathrm{b}}$ & {$[$ TCTA $] 5[$ TCTG $] 5[$ TCTA $] 3$ TA [TCTA]3 TCA [TCTA]2 TCCATA [TCTA]9 } & 27 & 0.010 & 0.000 & 0.032 \\
\hline 14 & {$[$ TCTA $] 4[$ TCTG 6 [TCTA]3 TA [TCTA]3 TCA [TCTA]2 TCCATA [TCTA]10 } & 28 & 0.135 & 0.152 & 0.190 \\
\hline 17 & [TCTA]4 [TCTG]6 [TCTA]3 TA [TCTA]3 TCA [TCTA]2 TCCATA [TCTA]11 & 29 & 0.163 & 0.185 & 0.204 \\
\hline $7 / 5$ & [TCTA]6 [TCTG]5 [TCTA]3 TA [TCTA]3 TCA [TCTA]2 TCCATA [TCTA]10 & 29 & 0.067 & 0.054 & \\
\hline 1 & [TCTA]6 [TCTG]5 [TCTA]3 TA [TCTA]3 TCA [TCTA]2 TCCATA [TCTA]7 TCA [TCTA]3 & 29.3 & 0.010 & 0.011 & - \\
\hline 17 & [TCTA]6 [TCTG]5 [TCTA]3 TA [TCTA]3 TCA [TCTA]2 TCCATA [TCTA]11 & 30 & 0.163 & 0.185 & 0.237 \\
\hline $9 / 7$ & [TCTA]4 [TCTG]6 [TCTA]3 TA [TCTA]3 TCA [TCTA]2 TCCATA [TCTA]12 & 30 & 0.087 & 0.076 & \\
\hline $4 / 2$ & [TCTA $] 5[$ TCTG]6 [TCTA]3 TA [TCTA]3 TCA [TCTA]2 TCCATA [TCTA]11 & 30 & 0.038 & 0.022 & \\
\hline 1 & [TCTA]7 [TCTG]5 [TCTA]3 TA [TCTA]3 TCA [TCTA]2 TCCATA [TCTA]10 & 30 & 0.010 & 0.011 & \\
\hline 3 & [TCTA]5 [TCTG]6 [TCTA]3 TA [TCTA]3 TCA [TCTA]2 TCCATA [TCTA]10 TA TCTA & 30.2 & 0.029 & 0.033 & 0.034 \\
\hline 2 & [TCTA]5 [TCTG]5 [TCTA]3 TA [TCTA]3 TCA [TCTA]2 TCCATA [TCTA]11TA TCTA & 30.2 & 0.019 & 0.022 & \\
\hline 2 & [TCTA]6 [TCTG]5 [TCTA]3 TA [TCTA]3 TCA [TCTA]2 TCCATA [TCTA]12 & 31 & 0.019 & 0.022 & 0.098 \\
\hline 1 & [TCTA]4 [TCTG]7 [TCTA]3 TA [TCTA]3 TCA [TCTA]2 TCCATA [TCTA]12 & 31 & 0.010 & 0.011 & \\
\hline 1 & [TCTA]5 [TCTG]6 [TCTA]3 TA [TCTA]3 TCA [TCTA]2 TCCATA [TCTA]12 & 31 & 0.010 & 0.011 & \\
\hline 6 & [TCTA]5 [TCTG]6 [TCTA]3 TA [TCTA]3 TCA [TCTA]2 TCCATA [TCTA]11 TA TCTA & 31.2 & 0.058 & 0.065 & 0.079 \\
\hline 1 & [TCTA]5 [TCTG]6 [TCTA]3 TA [TCTA]3 TCA [TCTA]2 TCCATA [TCTA]13 & 32 & 0.010 & 0.011 & 0.014 \\
\hline $6 / 3$ & [TCTA]5 [TCTG]6 [TCTA]3 TA [TCTA]3 TCA [TCTA]2 TCCATA [TCTA]12 TA TCTA & 32.2 & 0.058 & 0.033 & 0.024 \\
\hline $9 / 8$ & [TCTA]5 [TCTG]6 [TCTA]3 TA [TCTA]3 TCA [TCTA]2 TCCATA [TCTA]13 TA TCTA & 33.2 & 0.087 & 0.087 & 0.024 \\
\hline 1 & [TCTA]5 [TCTG]6 [TCTA]3 TA [TCTA]3 TCA [TCTA]2 TCCATA [TCTA]14 TA TCTA & 34.2 & 0.010 & 0.011 & 0.001 \\
\hline $1 / 0^{\mathrm{b}}$ & $[$ TCTA $] 11[$ TCTG $] 5$ [TCTA $] 3$ TA [TCTA]3 TCA [TCTA $] 2$ TCCATA [TCTA] 12 & 36 & 0.010 & 0.000 & - \\
\hline
\end{tabular}

a Allele frequencies of all samples were compared with allele frequencies of samples from Europeans and with allele frequencies of the in-house database.

b Allele only observed in individuals with African origin. 

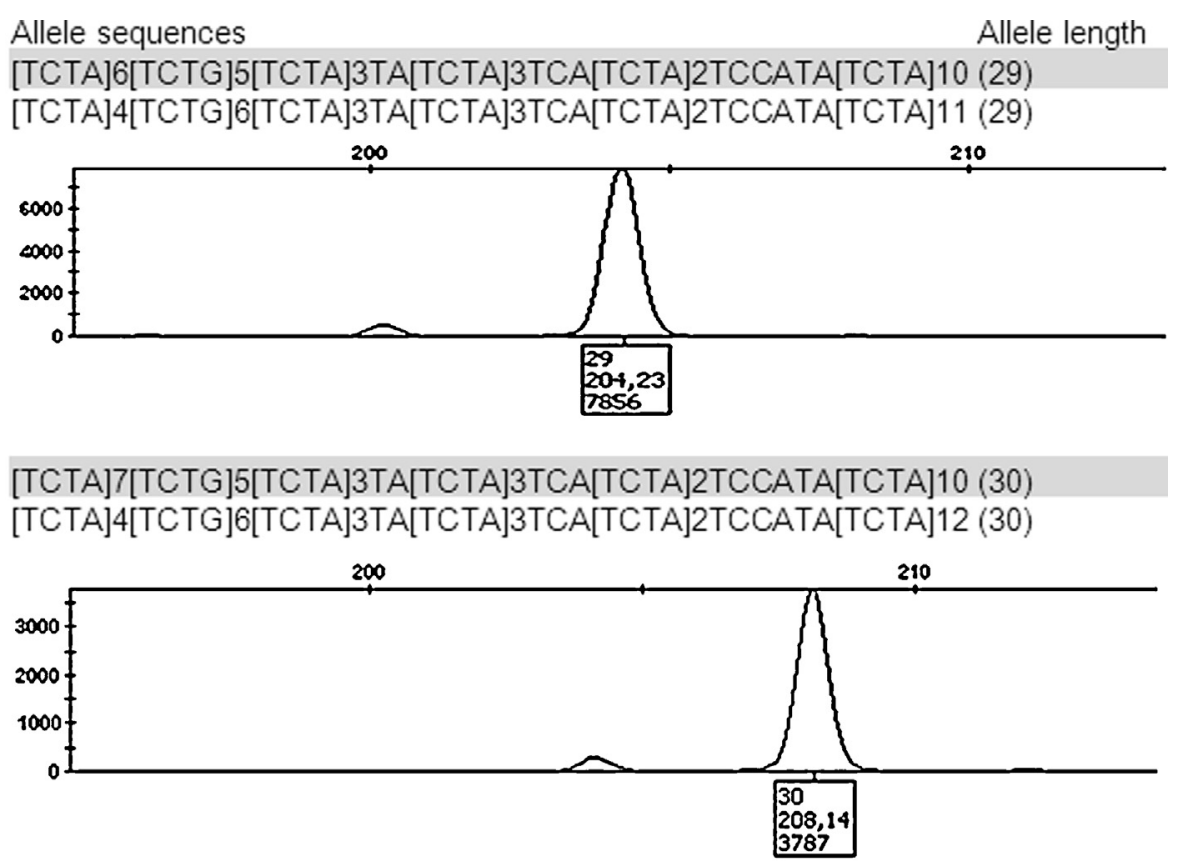

[TCTA]5[TCTG]6[TCTA]3TA[TCTA]3TCA[TCTA]2TCCATA[TCTA]11 (30)

[TCTA]4[TCTG]6[TCTA]3TA[TCTA]3TCA[TCTA]2TCCATA[TCTA]12 (30)

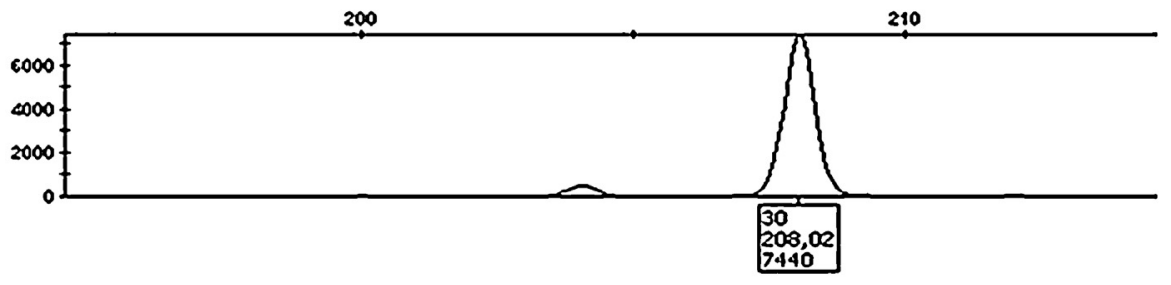

[TCTA]5[TCTG]6[TCTA]3TA[TCTA]3TCA[TCTA]2TCCATA[TCTA]11 (30)

[TCTA]4[TCTG]6[TCTA]3TA[TCTA]3TCA[TCTA]2TCCATA[TCTA]12 (30)

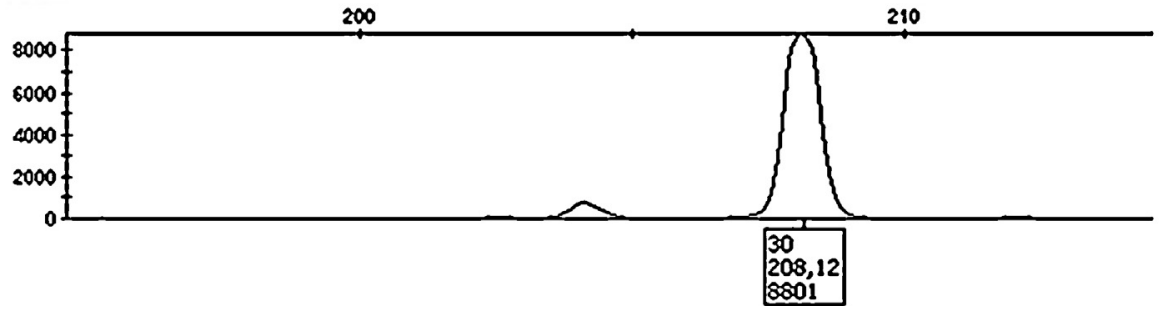

Fig. 1. DNA sequences and CE results in four unrelated individuals reported as homozygous by CE, but with different sequence composition as shown with NGS.

deletion) is under debate [12,20,27]. Our results supported the theory that insertions are favored over deletions in alleles with low numbers of uninterrupted repeat units. In this work, the mutations happened in alleles with 10-14 uninterrupted repeats (S4). Our results also support the view that mutations often occur in the longest uninterrupted repeat section [9] and that paternal mutations are more frequent compared to maternal ones.

The allele frequencies of the sequenced D21S11 loci in this study corresponded well with our in-house allele frequencies (see Table 2). Six of the 53 samples originated from unrelated

Table 3

Sub-repeat patterns of unrelated individuals.

\begin{tabular}{llllll}
\hline No. [TCTA]a & No. of alleles all/Europeans & No. [TCTG]b & No. of alleles all/Europeans & No. [TCTA]f & No. of alleles all/Europeans \\
\hline 4 & $41 / 39$ & 5 & $32 / 28$ & $9^{\text {a }}$ & $1 / 0$ \\
5 & $34 / 27$ & 6 & $71 / 63$ & 10 & $26 / 24$ \\
6 & $27 / 25$ & 7 & $1 / 1$ & 11 & $46 / 44$ \\
7 & $1 / 1$ & & & 13 & $20 / 14$ \\
$11^{\text {a }}$ & $1 / 0$ & & & 14 & $10 / 9$ \\
& & & & $1 / 1$ & \\
\hline
\end{tabular}

\footnotetext{
${ }^{\text {a }}$ Sub-repeat only observed in individuals with African origin.
} 
individuals with other ethnic background than Danish. Two alleles from individuals of African origin had a different sub-repeat composition compared to those of the rest of the sequenced alleles (see Tables 2 and 3). This may indicate a difference in the subrepeat patterns between Europeans and Africans. This observation will be explored further in our laboratory since more sequence data is needed to draw any conclusion.

The most interesting observation in this study was the identification of different sub-repeat compositions of several D21S11 alleles of identical lengths. This made it possible to identify the parental alleles that had mutated, and it made it possible to distinguish between individuals with alleles of the same lengths. This is best illustrated by the four apparently homozygous individuals that turned out to be heterozygous for allele 29 and 30 (Fig. 1). These results demonstrated the power of NGS in forensic genetic testing. Characterization of a mutation event in an autosomal STR may be essential in a relationship case. More identifiable alleles mean more statistical power of the STR investigation and essentially reduce the necessary number of loci that needs to be typed to solve a case to a certain level. It may also be easier to resolve DNA mixtures in crime case investigations, when alleles that appear identical in CE-analyses may be further characterized by NGS.

About half of the STRs selected for the European Standard Set and the CODIS set are simple STR repeats with only one repeat unit and little sequence variation except for the variation in number of repeats. Therefore, the full power of NGS can only be exploited by also including new complex STRs. NGS provides a possibility for constructing an all-in-one multiplex with relevant forensic markers that include, e.g. STRs, SNPs, indels and mtDNA markers. The use of NGS in forensic genetics will be an important research area in the near future and could offer the first real alternative to PCR-CE analysis.

\section{Acknowledgements}

We thank Thomas Berg for critical review of study proposal. We thank Maibritt Sigvardt, Anja Jørgensen and Nadia Jochumsen for technical assistance and Sarah Fordyce for constructive help with the manuscript.

\section{References}

[1] S.L. Fordyce, M.C. Avila-Arcos, E. Rockenbauer, C. Borsting, R. Frank-Hansen, F.T Petersen, E. Willerslev, A.J. Hansen, N. Morling, M.T. Gilbert, High-throughput sequencing of core STR loci for forensic genetic investigations using the Roche Genome Sequencer FLX platform, Biotechniques 51 (2011) 127-133.

[2] D.M. Bornman, M.E. Hester, J.M. Schuetter, M.D. Kasoji, A. Minard-Smith, C.A. Barden, S.C. Nelson, G.D. Godbold, C.H. Baker, B. Yang, J.E. Walther, I.E. Tornes, P.S. Yan, B. Rodriguez, R. Bundschuh, M.L. Dickens, B.A. Young, S.A. Faith, Short-read, high-throughput sequencing technology for STR genotyping, Biotechniques 0 (2012) 1-6. http://dx.doi.org/10.2144/000113857.

[3] E. Rockenbauer, M.B. Holgersson, S.L. Fordyce, M.C.Á. Arcos, C. Børsting, A.J. Hansen, R. Frank-Hansen, E. Willerslev, M.T.P. Gilbert, N. Morling, Sequences of microvariant/"off-ladder" STR alleles, Forensic Sci. Int. Genet. 3 (2011) e204-e205.

[4] C. Van Neste, F. Van Nieuwerburgh, D. Van Hoofstat, D. Deforce, Forensic STR analysis using massive parallel sequencing, Forensic Sci. Int. Genet. 6 (2012) 810-818.

[5] N.J. Loman, C. Constantinidou, J.Z. Chan, M. Halachev, M. Sergeant, C.W. Penn, E.R. Robinson, M.J. Pallen, High-throughput bacterial genome sequencing: an embarrassment of choice, a world of opportunity, Nat. Rev. Microbiol. 10 (2012) 599-606.
[6] F. Pitterl, K. Schmidt, G. Huber, B. Zimmermann, R. Delport, S. Amory, B. Ludes, $\mathrm{H}$. Oberacher, W. Parson, Increasing the discrimination power of forensic STR testing by employing high-performance mass spectrometry, as illustrated in indigenous South African and Central Asian populations, Int. J. Legal Med. 124 (2010) 551-558.

[7] J.V. Planz, K.A. Sannes-Lowery, D.D. Duncan, S. Manalili, B. Budowle, R. Chakraborty, S.A. Hofstadler, T.A. Hall, Automated analysis of sequence polymorphism in STR alleles by PCR and direct electrospray ionization mass spectrometry, Forensic Sci. Int. Genet. 6 (2012) 594-606.

[8] A.C. de Souza Goes, E.F. de Carvalho, I. Gomes, D.A. da Silva, E.H. Gil, A. Amorim, L. Gusmao, Population and mutation analysis of 17 Y-STR loci from Rio de Janeiro (Brazil), Int. J. Legal Med. 119 (2005) 70-76.

[9] S.H. Kim, N.Y. Kim, S.B. Hong, N.S. Cho, J.J. Kim, M.S. Han, W. Kim, Genetic polymorphisms of $16 \mathrm{Y}$ chromosomal STR loci in Korean population, Forensic Sci. Int. Genet. 2 (2008) e9-e10.

[10] D.J. Ballard, C. Phillips, G. Wright, C.R. Thacker, C. Robson, A.P. Revoir, D.S. Court, A study of mutation rates and the characterisation of intermediate, null and duplicated alleles for 13 Y chromosome STRs, Forensic Sci. Int. 155 (2005) 65-70.

[11] L. Gusmao, P. Sanchez-Diz, F. Calafell, P. Martin, C.A. Alonso, F. Alvarez-Fernandez, C. Alves, L. Borjas-Fajardo, W.R. Bozzo, M.L. Bravo, J.J. Builes, J. Capilla, M. Carvalho, C. Castillo, C.I. Catanesi, D. Corach, A.M. Di Lonardo, R. Espinheira, E. Fagundes de Carvalho, M.J. Farfan, H.P. Figueiredo, I. Gomes, M.M. Lojo, M. Marino, M.F. Pinheiro, M.L. Pontes, V. Prieto, E. Ramos-Luis, J.A. Riancho, A.C. Souza Goes, O.A. Santapa, D.R. Sumita, G. Vallejo, L. Vidal Rioja, M.C. Vide, C.I. Vieira da Silva, M.R. Whittle, W. Zabala, M.T. Zarrabeitia, A. Alonso, A. Carracedo, A. Amorim, Mutation rates at Y chromosome specific microsatellites, Hum. Mutat. 26 (2005) $520-528$.

[12] E.M. Dauber, A. Kratzer, F. Neuhuber, W. Parson, M. Klintschar, W. Bar, W.R. Mayr, Germline mutations of STR-alleles include multi-step mutations as defined by sequencing of repeat and flanking regions, Forensic Sci. Int. Genet. 6 (2012) 381-386.

[13] T. Kojima, T. Yamamoto, T. Yoshimoto, K. Tamaki, X.L. Huang, H. Ohtaki, R. Uchihi, J.A. Armour, S. Chen, Y. Katsumata, Tetrameric short tandem repeat (STR) system D15S233 (wg1d1): sequencing and frequency data in the japanese and Chinese populations, Leg. Med. (Tokyo) 1 (1999) 119-126.

[14] P. Wiegand, H.R. Schneider, M. Schurenkamp, M. Kleiber, B. Brinkmann, Tetranucleotide STR system D8S1132: sequencing data and population genetic comparisons, Int. J. Legal Med. 111 (1998) 180-182.

[15] B. Brinkmann, M. Klintschar, F. Neuhuber, J. Huhne, B. Rolf, Mutation rate in human microsatellites: influence of the structure and length of the tandem repeat, Am. J. Hum. Genet. 62 (1998) 1408-1415.

[16] H. Ellegren, Heterogeneous mutation processes in human microsatellite DNA sequences, Nat. Genet. 24 (2000) 400-402.

[17] M. Muller, U. Sibbing, C. Hohoff, B. Brinkmann, Haplotype-assisted characterization of germline mutations at short tandem repeat loci, Int. J. Legal Med. 124 (2010) 177-182.

[18] X. Xu, M. Peng, Z. Fang, The direction of microsatellite mutations is dependent upon allele length, Nat. Genet. 24 (2000) 396-399.

[19] H. Ellegren, Microsatellite mutations in the germline: implications for evolutionary inference, Trends Genet. 16 (2000) 551-558.

[20] H. Ellegren, Microsatellites: simple sequences with complex evolution, Nat. Rev. Genet. 5 (2004) 435-445.

[21] C. Borsting, J.J. Sanchez, H.E. Hansen, A.J. Hansen, H.Q. Bruun, N. Morling, Performance of the SNPforID 52 SNP-plex assay in paternity testing, Forensic Sci. Int. Genet. 2 (2008) 292-300.

[22] C. Borsting, E. Rockenbauer, N. Morling, Validation of a single nucleotide polymorphism (SNP) typing assay with 49 SNPs for forensic genetic testing in laboratory accredited according to the ISO 17025 standard, Forensic Sci. Int. Genet. 4 (2009) 34-42.

[23] M.C. Kline, C.R. Hill, A.E. Decker, J.M. Butler, STR sequence analysis for characterizing normal, variant, and null alleles, Forensic Sci. Int. Genet. 5 (2011) 329-332.

[24] C. Borsting, J.J. Sanchez, N. Morling, Forensic genetic DNA typing with PCR-based methods, in: S. Hughes, A. Moody (Eds.), PCR - Method Express Series, Scion Publishing Ltd., Bloxham, UK, 2007, pp. 123-142.

[25] M. Stene, A. Buchard, C. Børsting N. Morling Validation of the AmpFISTR ${ }^{\mathbb{R}}$ Identifiler ${ }^{\mathbb{R}}$ Direct PCR Amplification kit in a laboratory accredited according to the ISO17025 standard, Forensic Sci. Int. Genet. 3 (2011) e165-e166.

[26] A. Sajantila, M. Lukka, A.C. Syvanen, Experimentally observed germline mutations at human micro- and minisatellite loci, Eur. J. Hum. Genet. 7 (1999) 263-266.

[27] A.C. Mardini, R. Rodenbusch, S. Schumacher, F.G. Chula, C.T. Michelon, A.Z. Gastaldo, L.P. Maciel, S.E. de Matos Almeida, C.M. da Silva, Mutation rate estimates for 13 STR loci in a large population from Rio Grande do Sul, Southern Brazil, Int. J. Legal Med. (2011), http://dx.doi.org/10.1007/s00414-011-0642-x. 